disease. The SWE may be a useful tool for elucidation of early stage pathological changes of the SG when salivary gland functions are not impaired in SS.

Disclosure of Interest: None declared

DOI: 10.1136/annrheumdis-2018-eular.2589

\section{FRI0381 ARE THE ANTIPHOSPHOLIPID ANTIBODIES A “NEW" INDEPENDENT RISK FACTOR FOR ACCELERATED ATHEROSCLEROSIS}

N. Stoilov ${ }^{1}$, V. Boyadzhieva ${ }^{1}$, R. Rashkov ${ }^{1}$, S. Stefanov ${ }^{2} .{ }^{1}$ Clinic of Rheumatology, Umbal "Sv. Ivan Rilski", ${ }^{2}$ Clinic of Rheumatology, SBALDB, Sofia, Bulgaria

Background: The physiological function of phospholipids and their protein cofactors, which are aPL target, are well known. Beta 2 glycoprotein 1 is an impor tant transport molecule that binds negatively charged particles to free circulation, including various lipoproteins, such as LDL. In this context, $\beta 2 \mathrm{GPI}$ plays the role of protein co-factor of low density lipoprotein and is a key factor affecting serum levels of free LDL and total cholesterol, respectively. Its physiological function is suppressed by antibodies against it.

Objectives: We investigated the incidence of atherosclerosis in patients with antiphospholipid syndrome compared with systemic lupus erythematosus without $\mathrm{aPL}$ and healthy controls at pre-treatment sites.

Methods: We examined 219 people devided in three groups - APS (136), SLE, ${ }^{42}$ healthy controls. ${ }^{41}$

We performed an ultrasound examination of the carotid arteries, measuring intima-media thicknes as well and evaluate the vessel wall for atherosclerotic plaques.

We performed calcium score on the coronary arteries, aorta and aortic valve as well.

Results: Between our groups we do not foud statistically significant differece for BMI.

Comparative analysis for LDL-cholesterol showed higher APS median values ( $3.13 \mathrm{mmol} / \mathrm{L})$ compared to other groups. Using the Kruskal-Wallis test, we have a statistically significant result for a direct correlation with the antiphospholipid syndrome $(p=0.001)$. Patients with APS shows higher levels for LDL.

With the Kruskall-Wallis analysis, we found a statistically significant difference in IMT between the study groups for left $(p=0.005)$ and right $(p=0.014)$ carotid arteries.

With Mann-Whitney analysis, we do not detect statistically significant differences in IMT values between patients with primary antiphospholipid syndrome and those with secondary.

We investigated the frequency distribution of atherosclerotic plaques in individuals in the groups. In the right carotid artery, plaque was found in $18(31.6 \%)$ patients with APS, $3(7.3 \%)$ cases were recorded in healthy controls, and in the SLE group there were no reported cases. We establish a statistically significant correlation of carotid plaques in APS oatients $(p \leq 0.001)$

In our study group of patients with APS is characterised by the highest incidence of positive CaScore, and its maximum values are significantly higher than the other two groups. The maximum Agatson score for patients with antiphospholipid syndrome is $908 \mathrm{HU}$, with SLE being $2.1 \mathrm{HU}$, with healthy controls $-233 \mathrm{HU}$.

We ivestigate the levels of anti-ox LDL antibodies and we found that there is a correlation between the levels of aPL and anti-oxLDL.

Conclusions: The conclusion from our study is that the aPL could cosidered for independent risk factor for accelerated atherosclerosis. The aPL are responsible for dyslipidemia in some cases blocking the transport molecules like b2GPI.

Disclosure of Interest: None declared

DOI: 10.1136/annrheumdis-2018-eular.6776

\section{FRI0382 $\quad$ A STUDY OF MCP1 AND LIPOCALIN AS URINARY BIOMARKERS IN LUPUS NEPHRITIS}

R. Ramamoorthy, T.N. Tamilselvam, B. Kumar. Institute of Rheumatology, Madras Medical College, Chennai, India

Background: Currently, major efforts have been undertaken to identify biomarkers that can predict impending lupus renal flare, development of chronic kidney disease or reflect renal histology at the time of the flare.

Objectives: This study aims to assess the correlation of urinary biomarkers MCP1 and NGAL with the disease activity in lupus nephritis.

Methods: This was a prospective study conducted in a tertiary care centre. 60 patients with SLE were recruited. They were divided into 3 groups, one with Active Lupus Nephritis $(n=22)$, another with Inactive Lupus Nephritis $(n=20)$ and a third formed of SLE patients with no renal involvement $(n=18)$. For comparison another group of age and sex matched controls was taken $(n=20)$. Disease activity was correlated with disease activity indices, baseline characteristics and biopsy. Urinary MCP1 and NGAL were measured. Statistical analysis using SPSS11.5 was done to find the correlation between levels of urinary biomarkers and disease activity.

Results: In patients with active LN, both UMCP1/Cr and UNGAL/Cr were significantly elevated $(92.78,76.11 \mathrm{pg} / \mathrm{ml}, \mathrm{p}<0.001)$. In both control group and SLE without renal involvement the values of UMCP1/Cr and UNGAL/Cr were norma $(24.44,22.22 \mathrm{pg} / \mathrm{ml}$ in control and $24.3,22.80 \mathrm{pg} / \mathrm{ml}$ in SLE without renal involvement). In patients with inactive LN the values of UMCP1/Cr and UNGAL/Cr were observed to be significantly higher than control $(44.18,38.45 \mathrm{pg} / \mathrm{ml}, \mathrm{p}<0.005)$ and lower than those of active LN. Values of UMCP1/Cr and UNGAL/Cr were found to be in close correlation with mean rSLEDAl scores of active $L N^{10}$ and inactive $L N$ (3.6).

Conclusions: Levels of urinary biomarkers UMCP1 and UNGAL were significantly elevated in active lupus nephritis and found to have excellent correlation with disease activity index and rSLEDAI scores.

\section{REFERENCES:}

[1] Susianti $H$, Iriane VM, Dharmanata $S$, Handono K, Widijanti A, Gunawan A, Kalim H. Analysis of urinary TGF- 31 , MCP-1, NGAL, and IL-17 as biomarkers for lupus nephritis. Pathophysiology 2015 Mar;22(1):65-71. doi:10.1016/j.pathophys.2014.12.003. [Epub 2015 Jan 6].

[2] Susianti $\mathrm{H}$, et al. Urinary Neutrophil Gelatinase associated Lipocalin to monitor Lupus Nephritis Disease Activity. Biomark insights 2015;10:80-87.

Disclosure of Interest: None declared

DOI: 10.1136/annrheumdis-2018-eular.2184

\section{FRI0383 CHANGES IN WHITE MATTER MICROSTRUCTURE CORRELATE WITH SF-36 MENTAL COMPONENT SUBSCORE IN INFLAMMATORY NPSLE}

$\underline{\text { R.C. Monahan }}^{1}$, L. Beaart- van de Voorde ${ }^{1}$, C. Magro-Checa ${ }^{1}$, T. Huizinga ${ }^{1}$, H. Middelkoop ${ }^{2}$, M. van Buchem ${ }^{3}$, I. Ronen ${ }^{3}$, A. Kaptein ${ }^{4}$, G. Steup-Beekman ${ }^{1}$. ${ }^{1}$ Rheumatology; ${ }^{2}$ Neurology; ${ }^{3}$ Radiology; ${ }^{4}$ Psychiatry, Leiden University Medical Center, Leiden, Netherlands

Background: The diagnosis and treatment of neuropsychiatric systemic lupus erythematosus (NPSLE) is challenging due to the lack of a diagnostic gold standard and controlled trials. Immunosuppressive therapy is prescribed for immunemediated NPSLE manifestations, despite little evidence supporting its effectivity There is increasing evidence for the value of magnetization transfer imaging (MTI) in NPSLE. Recent research has demonstrated that white matter (WM) magnetic transfer ratio histogram peak height (MTR-HPH), a derived-MTI index, is a valuable radiological biomarker for the diagnosis and follow-up in inflammatory NPSLE. ${ }^{1}$ It remains unknown whether the changes in WM MTR-HPH also correlate with the change in health-related quality of life (QoL) in these patients.

Objectives: To analyse the correlation between change in WM MTR-HPH and change in QoL in different subsets of NPSLE (inflammatory, ischaemic, nonNPSLE).

Methods: Patients that visited the Leiden University Medical Centre (LUMC) NPSLE clinic between 2007 and 2015 were included in this study. The attribution process of NP events to SLE and one of its underlying pathogenic mechanisms (ischaemic or inflammatory) was established by multidisciplinary consensus. All patients underwent MRI examination at two different time points and filled in the Short-Form 36 (SF-36). Summary scores were deducted from the SF-36, leading to a Physical Component subscore (PCS) and a Mental Component subscore (MCS) for QoL. Spearman rank correlation coefficient (SR) was used to correlate WM MTR-HPH and the overall difference in change between visits in MCS and PCS. Data was analysed per patient and per event.

Results: A total of 15 patients (mean age $39.5 \pm 14.1$ years, $93 \%$ female) were included. Thirty-one NPSLE events were present, of which $68 \%$ were inflammatory, $13 \%$ ischaemic and $19 \%$ non-NPSLE events. Average time between visits was $2.9 \pm 1.5$ years. The per patient analysis showed statistical significant correlation between WM MTR-HPH and MCS change $(S R=0.62 ; p=0.01)$ and no effect on PCS change $(S R=0.09 ; p=0.76)$. The per event analysis showed a significant correlation between change in WM MTR-HPH and MCS in inflammatory events $(S R=0.56 ; p=0.01)$, but not in ischaemic $(p=0.20)$ or non-NPSLE events $(p=0.65)$ No correlation was observed between change in WM MTR-HPH and PCS in any of the subsets ( $p=0.21, p=0.40$ and $p=0.91$ respectively).

Conclusions: We are the first to demonstrate a correlation between a radiological biomarker and change in the mental component of QoL in inflammatory NPSLE patients. Our study supplies evidence for the use of immunosuppressive therapy in inflammatory NPSLE and supports the use of this radiological biomarker as an outcome measure for future trials in NPSLE. 